\section{Análise comparativa dos padrões de deglutição de crianças com paralisia cerebral e crianças normais}

\author{
Sandra R. Aurélio', Kátia F. Genaro', \\ Evaldo D. Macedo Filho ${ }^{3}$
}

Resumo

bjetivo: o propósito desse estudo foi comparar os padrões de deglutição em crianças com paralisia cerebral (PC) e crianças sem distúrbios neurológicos, relacionando a disfunção motora oral, a quadriplegia e a consistência dos alimentos com o tempo de deglutição. Material e método: Setenta e seis crianças participaram desse estudo, com idades variando de 1 a 5 anos, sendo 57 com paralisia cerebral e 19 sem distúrbios neurológicos. Três consistências de alimentos foram examinadas: líquida (suco), pastosa (iogurte) e sólida (bolacha), sendo cronometrado o tempo gasto para deglutir cada uma delas, durante o horário normal de lanche das mesmas. Resultados: Os resultados mostraram que: 1) o grupo com PC levou até 14,2 vezes mais tempo para deglutir os alimentos que o outro grupo; 2) quanto maior era a disfunção motora oral das crianças, maior era o tempo gasto para deglutir os alimentos e, 3) das três consistências pesquisadas, o alimento pastoso foi aquele, em que os tempos de deglutição obtidos de cada grupo estiveram mais próximos.

\section{Comparative analysis of swallowing patterins between children with cerebral palsy and normal children}

Palavras-chave: deglutição, alimentação, paralisia cerebral. Key words: swallowing, feeding, cerebral palsy.

\section{/ Summary}

\footnotetext{
Mestre em Distúrbios da Comunicação Humana.

Doutora em Distúrbios da Comunicação Humana.

${ }^{3}$ Doutor em Cirurgia.
}

Instituiç̃o: Universidade Tuiuti do Paraná

Endereço para correspondência: Sandra Regina Vasconcelos - R. Verônica Szeremeta, 23 - São Brás Curitiba - PR - CEP 82320-410

Tel: (0xx41) 272-6792 ou (0xx41) 9977-3129 - E-mail: jlvasco@uol.com.br

Apresentado como Tema Livre no III Encontro Brasileiro de Disfagia.

Artigo recebido em 30 de novembro de 2001. Artigo aceito em 20 de dezembro de 2001. 


\section{INTRODUÇÃO}

Os avanços tecnológicos e médicos nas três últimas décadas têm aumentado as chances de vida de crianças pré-termo e de baixo peso, surgindo, então, novos desafios para os profissionais que atuam com essas crianças. Algumas delas, em decorrência de problemas durante o nascimento, acabam desenvolvendo problemas neurológicos como é o caso da paralisia cerebral ${ }^{1}$.

Sabe-se que de 39\% a 56\% das crianças com problemas crônicos do desenvolvimento, dentre eles a paralisia cerebral, apresentam ou irão desenvolver um distúrbio da deglutição ${ }^{2}$. As conseqüências desse distúrbio acabam por acarretar novos problemas de saúde que, por sua vez, pioram ainda mais as condições globais desses indivíduos e sua capacidade de adaptar-se socialmente.

A dificuldade motora básica dessas crianças pode afetar a função motora oral (FMO), entendida como os aspectos motores e sensoriais das estruturas da cavidade oral e faringe até a entrada do esôfago ${ }^{3}$. Por esta razão, sabemos que essas crianças pertencem a um grupo de risco para desenvolver desordens da deglutição ou disfagia, o que pode ser observado como um dos primeiros sinais de seu distúrbio neurológico.

As desordens da função motora oral podem gerar um espectro de incapacidades em cada etapa do processo de deglutição com potenciais interações simultâneas. Em um número significativo de crianças, as alterações crônicas da deglutição resultam em desnutrição, desidratação, aspiração e pneumonia.

Os sintomas usualmente observados nesses casos são regurgitação, dificuldade de deglutir a própria saliva, tosse no momento da alimentação e instabilidade respiratória ou apnéia, dentre outros, que podem gerar aversão para comer determinados alimentos, grande tempo despendido com a alimentação e a necessidade de dieta especial, diferente da utilizada pelo restante da família. Estes sintomas acabam gerando uma situação de tensão e ansiedade nos pais, o que torna o ato de comer difícil, cansativo e de pouco prazer.

Poucos estudos de curta duração sobre a expectativa de vida de pessoas com paralisia cerebral nesta área sugerem que os fatores mais importantes na definição da expectativa de vida desses indivíduos são: a presença de retardo mental severo e a mobilidade corporal reduzida, somadas à dificuldade de se alimentar.

A utilização de técnicas específicas para se estabelecer um claro diagnóstico sobre o distúrbio da deglutição contribui para o desenvolvimento de protocolos de tratamento criativos e realistas. A observação desta função colabora para a previsão de um desenvolvimento sadio e uma melhor qualidade de vida das crianças com paralisia cerebral.

Tendo em vista a importância da alimentação para a vida dos indivíduos e da dificuldade que as crianças com paralisia cerebral podem apresentar para deglutir, o presente trabalho tem por objetivo caracterizar o padrão de deglutição de crianças com paralisia cerebral, comparativamente a um grupo de crianças normais, relacionando o grau de disfunção motora oral, a quadriplegia e a consistência do alimento com o tempo gasto na deglutição.

\section{MATERIAL E MÉTODO}

Esse estudo foi realizado em dois grupos de crianças com faixa etária entre 1 e 5 anos, residentes na cidade de Curitiba, sendo um grupo (GI) formado por 57 crianças portadoras de paralisia cerebral, procedentes de escolas especiais e outro grupo (GII) formado por 19 crianças sem distúrbios neurológicos, alunos de escola regular. Das 57 crianças do GI, 27 eram do gênero feminino e 30 do gênero masculino e das 19 crianças do GII, 10 eram do gênero masculino e 9 gênero feminino.

No grupo de crianças com paralisia cerebral, 32 eram quadriplégicas, 15 diplégicas e 10 hemiplégicas. Das crianças diplégicas e hemiplégicas, todas $(n=35)$ eram espásticas. Dentre as 32 crianças quadriplégicas, 62,5\% $(n=20)$ eram espásticas; $9,3 \%(n=3)$ possuíam atetose; $6,2 \%$ $(n=2)$ eram flácidas e $21,8 \%(n=7)$ possuíam o tipo misto da paralisia cerebral. O grau do comprometimento variou entre leve a severo.

Para a realização do exame foi elaborado um protocolo de avaliação dos padrões de deglutição, o qual foi aplicado em todas as crianças sob as mesmas condições, tomando-se o cuidado de observar o horário natural de lanche das crianças para aplicar o protocolo, garantindo que elas estivessem com fome, de forma a evitar distorções nos resultados.

As crianças eram posicionadas sentadas em cadeiras adaptadas, quando necessário, de forma a ficarem confortáveis e em postura estável, sendo que algumas crianças receberam ajuda adicional do professor para controle da postura. Um pequeno número de crianças precisou ser alimentado no colo, devido a presença de reflexos primitivos, sendo essa a única forma de adaptação confortável para a alimentação.

O material para aplicação do protocolo foi convencionado como sendo: $100 \mathrm{ml}$ de suco de laranja (alimento líquido), 140 gramas de iogurte de morango homogêneo e sem pedaços de frutas (alimento pastoso) e 12 gramas de bolacha recheada de chocolate (alimento sólido), os quais foram ingeridos em sua totalidade e na ordem apresentada. Foi utilizado, ainda, cronômetro marca Technos com medidas em minutos e segundos para se mensurar os tempos de deglutição de cada tipo de alimento.

O protocolo de investigação constou da identificação e do resultado da observação da presença de sete comportamentos relacionados à função motora oral (FMO), sendo eles: reflexo de mordida, controle labial, sialorréia, 
capacidade de tragar líquido do copo, capacidade de retirar o alimento pastoso da colher, ocorrência de mastigação e condução do alimento e tempo gasto na deglutição de cada uma das três consistências de alimento pesquisadas.

Para analisar os resultados obtidos foi elaborada uma escala, atribuindo-se valor 1 para comportamentos considerados mais maduros ou 0 para comportamentos considerados mais imaturos, aplicada para cada um dos 7 itens da FMO (Quadro 1).

Cada criança recebeu uma pontuação final que correspondeu à soma dos valores dos sete itens da FMO, de forma que esta pontuação variou de 0 a 7 pontos, demonstrando o desempenho de cada criança para a FMO, estando ela mais próxima do normal quanto mais próximo de 7 estiver o total.

Quadro 1. Itens considerados na função motora oral (FMO) e os valores atribuídos.

\begin{tabular}{|lcc|}
\hline \multicolumn{1}{|c}{ FMO } & 1 & 0 \\
\hline Reflexo de mordida & Ausente & Presente \\
Controle labial & Presente & Ausente \\
Sialorréia & Ausente & Presente \\
Tragar do copo & Presente & Ausente \\
Retirar da colher & Presente & Ausente \\
Mastigar & Presente & Ausente \\
Condução & Normal & Anormal \\
\hline
\end{tabular}

Foram registrados os tempos gastos para deglutir cada um dos três tipos de alimentos, a partir da introdução da primeira porção na boca, até seu esvaziamento na última deglutição. Comparou-se os tempos medianos de deglutição de cada alimentos entre os dois grupos e a influência da quadriplegia. As crianças foram reagrupadas conforme o grau da disfunção motora oral: leve, moderada, severa e profunda ou FMO normal. Para cada um dos grupos, foi levantado o tempo mediano gasto na deglutição de cada uma das consistências avaliadas; assim, analisou-se a influência da disfunção motora oral nos tempos de deglutição. Por fim, relacionou-se as três consistências de alimento aos tempos de deglutição.

Os dados receberam tratamento estatístico, por meio do programa Sphinx Léxica versão 209K para levantamento dos porcentuais e cruzamento entre variáveis.

\section{RESULTADOS}

A primeira análise compara cada um dos 7 itens da função motora oral (FMO) entre os dois grupos, procurando-se ter uma idéia do padrão funcional dos grupos e observar suas diferenças e, em seguida, as crianças do GI são divididas em grupos de acordo com o grau da disfunção motora oral (DMO). Os tempos de deglutição foram associados à FMO normal, levemente, moderadamente, severamente e profundamente comprometida.

Podem ser notadas diferenças na ocorrência dos sete itens da FMO observados entre os grupos e para nenhum dos itens os dois grupos se equipararam. A capacidade de controlar os lábios evitando o escape do bolo alimentar para fora da cavidade oral durante a deglutição foi o item em que o GI e GII mais se distanciaram em termos de ocorrência. Já a habilidade de tragar o líquido do copo foi a que os dois grupos mais se aproximaram (Tabela 1).

As habilidades mais maduras foram observadas menos freqüentemente nas crianças quadriplégicas e os comportamentos considerados mais maduros ocorreram menos freqüentemente. Todas as crianças que apresentaram reflexo de mordida eram quadriplégicas.

A deglutição normal ocorreu associada à ausência do reflexo de mordida em 93\% dos casos, a presença da capacidade de tragar do copo em 90\%, a capacidade de retirar o alimento da colher em $79 \%$ e com a capacidade de mastigar em 86\%. Já a condução anormal ocorreu associada à ausência do controle labial em 96\% dos casos e a presença

Tabela 1. Distribuição da amostra segundo a presença dos itens da FMO por grupo.

\begin{tabular}{|c|c|c|c|c|c|c|c|}
\hline Grupo & Reflexo de mordida & Controle labial & Sialorréia & Tragar do copo & Retirar da colher & Mastigar & Conduzir \\
\hline $\begin{array}{c}\text { Gl } \\
(57)\end{array}$ & $\begin{array}{l}24 \% \\
(14) \\
\end{array}$ & $\begin{array}{l}28 \% \\
(16) \\
\end{array}$ & $\begin{array}{l}63 \% \\
(36) \\
\end{array}$ & $\begin{array}{l}73 \% \\
(42) \\
\end{array}$ & $\begin{array}{c}54 \% \\
(31) \\
\end{array}$ & $\begin{array}{l}70 \% \\
(40) \\
\end{array}$ & $\begin{array}{l}50 \% \\
(29) \\
\end{array}$ \\
\hline $\begin{array}{l}\text { Quadriplegico } \\
\text { (32) }\end{array}$ & $\begin{array}{l}44 \% \\
(14)\end{array}$ & $\begin{array}{l}9 \% \\
(2)\end{array}$ & $\begin{array}{l}90 \% \\
(28)\end{array}$ & $\begin{array}{l}44 \% \\
(14)\end{array}$ & $\begin{array}{c}28 \% \\
(8)\end{array}$ & $\begin{array}{l}53 \% \\
(16)\end{array}$ & $\begin{array}{c}19 \% \\
(6)\end{array}$ \\
\hline $\begin{array}{c}\text { Gll } \\
(19)\end{array}$ & $\begin{array}{l}0 \% \\
(0)\end{array}$ & $\begin{array}{c}100 \% \\
(19)\end{array}$ & $\begin{array}{l}0 \% \\
(0)\end{array}$ & $\begin{array}{c}100 \% \\
(19) \\
\end{array}$ & $\begin{array}{c}100 \% \\
(19)\end{array}$ & $\begin{array}{c}100 \% \\
(19) \\
\end{array}$ & $\begin{array}{c}100 \% \\
(19)\end{array}$ \\
\hline
\end{tabular}

Tabela 2. Correlação entre a condução normal e anormal e a ocorrência dos itens pesquisados da FMO.

\begin{tabular}{|c|c|c|c|c|c|c|}
\hline Condução & Reflexo de mordida & Controle labial & Sialorréia & Tragar do copo & Retirar da colher & Mastigar \\
\hline $\begin{array}{c}\text { Normal } \\
\text { (29) }\end{array}$ & $\begin{array}{l}7 \% \\
(2)\end{array}$ & $\begin{array}{l}52 \% \\
(15)\end{array}$ & $\begin{array}{c}34 \% \\
(9)\end{array}$ & $\begin{array}{l}90 \% \\
(26)\end{array}$ & $\begin{array}{l}79 \% \\
(22)\end{array}$ & $\begin{array}{l}86 \% \\
(24)\end{array}$ \\
\hline $\begin{array}{c}\text { Anormal } \\
\text { (28) }\end{array}$ & $\begin{array}{l}43 \% \\
(12)\end{array}$ & $\begin{array}{l}4 \% \\
(1)\end{array}$ & $\begin{array}{l}93 \% \\
(26)\end{array}$ & $\begin{array}{l}57 \% \\
(15)\end{array}$ & $\begin{array}{c}28 \% \\
(7)\end{array}$ & $\begin{array}{l}54 \% \\
(15)\end{array}$ \\
\hline
\end{tabular}


de sialorréia em 93\%. Das crianças que deglutiram de forma anormal (necessitando de posturas ou manobras facilitadoras), 90\% eram quadriplégicas (Tabela 2).

A seguir está apresentada a classificação da FMO das crianças do GI e GII, conforme pontuação dada a cada item da FMO. No grupo GI, 23\% das crianças apresentaram FMO normal, enquanto esse índice foi de 100\% no grupo GII. Dessa forma, $77 \%$ das crianças demonstraram algum grau de disfunção motora oral no grupo GI, variando entre leve e profunda.

As relações entre FMO e tempo de deglutição são mostradas na Tabela 3 e revelam que quanto maior a disfunção motora oral, maior o tempo gasto para realizar a deglutição em cada uma das três consistências de alimento, sugerindo que DMO e tempo de deglutição estão direta-

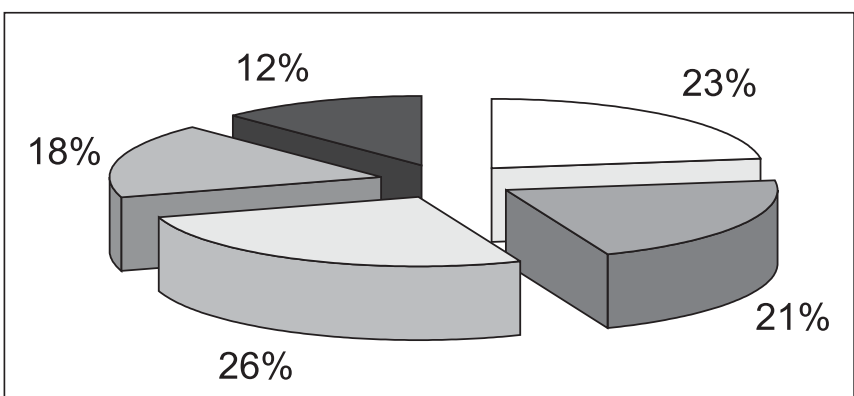

$\square$ Normal $\square$ Leve $\square$ Moderada $\square$ Severa $\square$ Profunda

Gráfico 1. Distribuição de gi segundo classificação da FMO.

mente relacionados.

Tabela 3. FMO, consistência do alimento e tempos medianos de deglutição em segundos por grupo.

\begin{tabular}{lcccc}
\hline Grupos & DMO & Líquido & Pastoso & Sólido \\
\hline GI & Normal & 78 & 115 & 200 \\
& Leve & 92 & 101,5 & 238 \\
& Moderada & 187 & 173 & 310 \\
& Severa & 352,5 & 264 & 506 \\
& Profunda & 413 & 479 & - \\
\hline Gll & Normal & 29 & 74 & 102
\end{tabular}

No grupo GI, 30\% das crianças apresentaram disfunção motora oral entre profunda (18\%) e severa (12\%), sendo todas quadriplégicas. Desta forma, as crianças que demoraram maior tempo para deglutir os alimentos eram as quadriplégicas. Das 32 crianças quadriplégicas, 53,1\% $(\mathrm{n}=17)$ apresentaram disfunção motora oral entre severa ou profunda.

Em relação à consistência do alimento e os tempos de deglutição, as crianças do GI com FMO profundamente comprometida levam 14,2 vezes mais tempo para deglutir alimentos líquidos e 6,4 vezes mais para o alimento pastoso do que as crianças de GII. Nenhuma das crianças com disfunção motora oral profunda deglutiu a consistência sólida. Das crianças com disfunção motora oral severa, apenas 30\% $(n=3)$ deglutiram a consistência sólida, as quais levaram 4,9 vezes mais tempo para deglutir o sólido que o GII. O alimento pastoso foi aquele em que os tempos de deglutição mais se aproximaram entre os dois grupos e o líquido, a consistência que se observou maior diferença dos tempos medianos de deglutição entre os dois grupos estudados. O alimento sólido foi o menos freqüentemente deglutido (Tabela 3).

\section{DISCUSSÃO}

Os problemas nutricionais são um desafio atual para quem atua com crianças com paralisia cerebral e a compreensão sobre a condição de saúde dessas crianças se faz necessária. Observa-se, nas publicações científicas, a tendência ao atendimento interdisciplinar no que diz respeito à deglutição. A integração com outros profissionais como a nutricionista, o gastroenterologista, o endoscopista, o pediatra, o fisioterapeuta, dentre outros, nos tem feito refletir acerca da atuação e do papel do fonoaudiólogo nessas equipes interdisciplinares.

O fonoaudiólogo deve contemplar a questão da saúde da criança com uma visão integral, observando a função motora oral dentro da perspectiva da boa nutrição e hidratação da criança por ele assistida. Isso equivale a dizer ter uma visão funcional da deglutição, observando se a capacidade motora de transportar o bolo alimentar e a eficácia para manter a boa nutrição da criança e colaborar no seu desenvolvimento, objetivo principal desse processo.

Foi com essa visão que se desenvolveu esse estudo, com o intuito de discutir questões relativas ao tempo de deglutição dessas crianças, e em última instância, uma reflexão acerca do tempo real gasto por elas durante as refeições diárias. A relevância desse questionamento baseia-se na necessidade de uma visão realista e objetiva em relação à deglutição, para preconizar-se melhores procedimentos e condutas a respeito da alimentação das mesmas.

A análise qualitativa da deglutição tem sido estudada por vários autores no que diz respeito às alterações de postura e movimento, quanto a capacidade de reduzir, formar e propelir o bolo alimentar, bem como o escape prematuro, penetração e aspiração de alimentos nas vias aéreas, entre outras. Esses estudos são de grande utilidade clínica, pois fornecem sustentação teórica para a prática diária com indivíduos que sofrem de problemas dessa natureza.

Parâmetros quantitativos, como o tempo do transporte do bolo em cada etapa do processo e o tempo gasto nas refeições, podem ser parâmetros igualmente importantes ${ }^{4,5,6}$.

Uma análise quantitativa deve ser considerada, especialmente para as crianças que possuem o distúrbio da função motora oral em conjunto com má nutrição, crescimento linear, perda de peso ou baixa imunidade, entre 
outras. A utilização de uma consistência de alimento de mais fácil deglutição deve ser preconizada nesses casos, antes que outras técnicas mais invasivas sejam necessárias, como por exemplo, a gastrostomia ${ }^{7,8,9,10}$.

Percebe-se que uma idéia do tempo despendido pela família para alimentar as suas crianças é ponto importante para que se decida a respeito das consistências de alimentos mais indicadas para cada criança, a forma de alimentá-las e a freqüência ${ }^{11,12,13}$. Estudos anteriores já indicam que as famílias tendem a gastar um tempo muito menor que o necessário para alimentar essas crianças, o que pode contribuir para uma diminuição da quantidade da ingesta deglutida e, usualmente, aumentar as chances de má nutrição e hidratação $0^{14,15}$

A observação da atividade reflexa anormal e da sialorréia tem sido objeto de muitos trabalhos sobre distúrbio da deglutição em crianças com paralisia cerebral, uma vez que acarretam transtornos na FMO e espelham o funcionamento imaturo do aparelho estomatognático. Da mesma forma, as habilidades de conter o bolo alimentar, tragar líquidos, retirar alimento da colher, mastigar e deglutir normalmente, relacionam-se diretamente com a especialização dos movimentos do trato aerodigestivo, desenvolvimento do sistema estomatognático e amadurecimento do sistema nervoso central. Essas habilidades tornam possível a manutenção da boa nutrição e o crescimento dos indivíduos ${ }^{3,16}$. Por essa razão, escolheu-se observar esses aspectos da FMO.

A alteração da deglutição em indivíduos com múltiplos impedimentos vem sendo relatada por vários autores, sob diferentes perspectivas. Reilly et al (1996) ${ }^{2}$ constataram em seu estudo que $38 \%$ da sua amostra apresentou deglutição alterada. Já em nosso estudo, observamos que $49 \%$ do grupo GI não foi capaz de realizar a condução normalmente, enquanto que todas as crianças do grupo GII o fizeram; as diferenças referem-se ao fato de que os autores levantaram dificuldades de deglutição referentes apenas ao primeiro ano de vida das crianças de sua amostra. Lanert \& Ekberg $(1995)^{13}$ relatam propulsão inadequada, especialmente nos quadriplégicos. Da mesma forma, em nosso estudo, houve relação entre a alteração da condução e a quadriplegia, uma vez que $90 \%$ das crianças com alteração da condução eram quadriplégicas. Wrigth et al (1996) ${ }^{17}$ estudaram crianças com paralisia cerebral severa e encontraram $75 \%$ de crianças com reflexo da deglutição atrasado. Park et al, (1992); ${ }^{18}$ Tawfiik et al (1997) ${ }^{19}$ associaram as dificuldades de condução à necessidade da gastrostomia como recurso para garantir a saúde dessas crianças.

Algumas correlações importantes foram feitas entre os comportamentos observados. A ocorrência de uma habilidade mais madura geralmente é acompanhada de outras. A correlação entre a deglutição normal e a grande porcentagem de crianças com ausência do reflexo de mordida, capacidade de retirar o alimento da colher, capacidade de tragar líquidos e a possibilidade da mastigação podem fornecer importante material de suporte a prática terapêutica. A prescrição de programas de tratamento incentivando o surgimento dessas habilidades possivelmente levará a ganhos quantitativos e qualitativos em relação à deglutição dessas crianças, melhora de sua condição nutricional e qualidade de vida.

A população com paralisia cerebral apresentou funcionamento variado em relação à deglutição, sendo as crianças quadriplégicas aquelas que mais necessitam de atenção. O esforço em dar condições para um desenvolvimento normal das funções orais para esse grupo, ou melhor, a tentativa de levar a criança da alimentação imatura (dieta homogênea e pastosa) para a alimentação madura (dieta heterogênea e sólida), deve ter o cuidado de contemplar incessantemente a boa condição de saúde dessas crianças.

Na nossa amostra de com paralisia cerebral, 77\% apresentou disfunção motora oral. Das crianças do GI, $30 \%$ tinham disfunção motora oral severa ou profunda. Nossos achados são similares aos de Reilly et al (1996), ${ }^{2}$ que encontraram $36,2 \%$ das suas crianças com disfunção motora oral severa e $90 \%$ de sua amostra com algum grau de disfunção motora oral. A diferença dos $90 \%$ para os $75 \%$ possivelmente se refere ao fato de que $70 \%$ da amostra de Reilly apresentou paralisia cerebral severa ou profunda, enquanto na nossa apenas $66 \%$ das crianças apresentou paralisia cerebral entre moderada e severa.

A disfunção motora oral moderada ou severa foi associada à quadriplegia, dados iguais aos de Reilly et al $(1996)^{2}$. No grupo quadriplégicos encontramos 97\% com disfunção motora oral, semelhante aos resultados de Morton et al $(1993)^{11}$ que referem $100 \%$ de seu grupo de quadriplégicos com disfunção motora oral e aos de Wrigth et al (1996) ${ }^{17}$ que citam 97\%. Nossos dados sugerem, ainda, que quanto maior a disfunção motora oral, maior o tempo gasto para alimentar essas crianças, como citam Jones $(1989)^{14}$; Morton et al (1993); Arvedson (1993) (1994); Lanert \& Ekberg (1995) ${ }^{13}$ e Buchholz (1997) ${ }^{20}$. Nesse sentido, o grupo de crianças quadriplégicas foi o com maior disfunção motora oral e, portanto, o que mais demorou a deglutir os alimentos, conseqüentemente, o que gasta maior tempo com a alimentação.

Nossos resultados indicam a existência de uma grande diferença entre o tempo gasto para alimentar crianças sem distúrbios neurológicos e crianças com paralisia cerebral, especialmente nas crianças quadriplégicas. Observamos que as crianças com paralisia cerebral podem levar até 14,2 vezes mais tempo para deglutir, comparativamente ao grupo sem distúrbios neurológicos. Lanert \& Ekberg $(1995)^{13}$ encontram um dado bastante semelhante ao nosso e declaram que as crianças podem gastar até 15 vezes mais tempo e levar até 7 horas por dia se alimentando. Jones (1989) ${ }^{14}$ afirma que algumas crianças 
podem gastar entre 4 a 6 horas nessa atividade, fato este que pode levar as famílias à frustração frente à dificuldade de alimentar essas crianças e ao estresse, colaborando para diminuição de oferta de alimento as mesmas e, conseqüente, má nutrição.

Das três consistências pesquisadas, o alimento líquido foi o que mostrou maiores diferenças de tempo para ser deglutido entre os grupos e o pastoso, o menor. A deglutição do alimento sólido foi menos freqüente que as outras consistências, mas quando ocorre, a diferença entre o tempo gasto pelos dois grupos é menor que para o alimento líquido. Essas diferenças foram observadas por outros pesquisadores. Gisel et al (1995) ${ }^{21}$ advertem que a consistência pastosa deve ser considerada para crianças com disfunção motora oral e desnutrição, uma vez que é mais fácil de ser manipulada e pode reduzir a tosse e a aspiração, facilitar a alimentação e a nutrição da criança. Helfrich-Miller et al (1986) ${ }^{22}$ indicam o uso do alimento sólido, associado à estimulação motora oral para o tratamento de crianças com paralisia cerebral, com o objetivo de diminuir o tempo de trânsito faríngeo, diminuir resíduos e a necessidade de múltiplas deglutições para limpar o conteúdo da faringe.

Nossos resultados sugerem a necessidade de monitorização das crianças com paralisia cerebral, especialmente as quadriplégicas, no que se refere a questões da deglutição e nutrição. O enorme tempo gasto durante as refeições, a reduzida quantidade de alimento, a disfunção motora oral, o crescimento e o aumento no gasto de energia, podem contribuir para uma estagnação do crescimento e prejuízos no desenvolvimento global da criança.

\section{CONCLUSÃO}

Por meio da aplicação do protocolo de avaliação dos padrões de deglutição em um grupo de crianças, 57 com paralisia cerebral e 19 sem distúrbios neurológicos, obteve-se as conclusões a seguir: quanto maior a disfunção motora oral, maior o tempo gasto para deglutir os alimentos nas três consistências pesquisadas, sendo o tempo de deglutição no grupo com paralisia cerebral até 14,2 vezes maior do que o grupo sem distúrbios neurológicos.

Das três consistências pesquisadas, o alimento líquido foi o que mais se afastou do grupo normal; no que diz respeito aos tempos de deglutição, o alimento pastoso foi o de menor diferença entre os grupos e o alimento sólido o menos freqüentemente deglutido pelo grupo com paralisia cerebral.

O grupo quadriplégico foi o de pior desempenho em relação a FMO e aos tempos de deglutição dos alimentos, sendo o que necessita de maior atenção no tocante às questões da deglutição.

\section{AGRADECIMENTO}

À escola Vivian Marçal, Associação Paranaense de Reabilitação - APR e à Clínica de Reabilitação da Fundação Pedro Seleme, por permitirem o uso de suas instalações para aplicação do Protocolo de Pesquisa. Às famílias e crianças envolvidas neste estudo, pela gentileza em participar da pesquisa.

\section{REFERÊNCIAS BIBLIOGRÁFICAS}

1. Brodsky L, Arvedson J. Introduction: Rationale for Interdisciplinary Care. In: ____ Pediatric Swallowing and Feeding: Assessment and Managment. San Diego California: Singular Publishing Group 1993:1-4.

2. Reilly S, Skuse D, Poblete X. Prevalence of feeding problems and oral motor dysfunction in children with cerebral palsy: a community survey. The Journal of Pediatrics 1996;129:877-82.

3. Arvedson J, Rogers B, Brodsky J. Anatomy, Embriology and Physiology. In: Arvedson JC, Brodsky L (Eds.). Pediatric Swallowing and Feeding: Assessment and Managment. San Diego California: Singular Publishing Group 1993:5-52.

4. Logemann JA. Swallowing Physiology and Phatophysiology. Otolaryngologic Clinics of North America Nov 1988;21(4):613-23.

5. Dantas RO, Dodds WJ. Influência da Viscosidade do Bolo Alimentar Deglutido na Motilidade da Faringe. Arquivo de Gastroenterologia out/dez. 1990;27(4):164-168.

6. Mcpherson KA, KENNY DJ, KOHEIL R et al. Ventilation and Swallowing Interactions of Normal Children and Children with Cerebral Palsy. Developmental Medicine and Child Neurology 1992;34:577-588.

7. Young C. Nutrition. In: Arvedson JC, Brodsky L (Eds.). Pediatric Swallowing and Feeding: Assessment and Managment. San Diego California: Singular Publishing Group 1993:157-208.

8. Amundson JA, Sherbondy A, Van Dyke DC, Alexander R. Early Identification and Treatment Necessary to Prevent Malnutrition in Children and Adolescents with Severe Disabilities. Journal of the American Dietetic Association Aug 1994;94(8):880-3.

9. Gisel EG, Applegate-Ferrante T, Benson JE, Bosma JF. Effect of Oral Sensorimotor Treatment on Measures of Growth, Eating Efficiency and Aspiration in the Dysphagic Child with Cerebral Palsy. Developmental Medicine and Child Neurology 1995;37:528543.

10. Bosma JF. Development and Impairments of Feeding in Infancy and Childhood. In: Groher ME. Dysphagia: Diagnosis and Management. $3^{\text {rd }}$ Edition, Butterworth-Heinemann 1997:131167.

11. Morton RE, Bonas R, Fourie B, Minford J. Videofluoroscopy in the Assessment of Feeding Disorders of Children with Neurological Problems. Developmental Medicine and Child Neurology 1993;35(5):388-395.

12. Rogers BT, Arvedson J, Msall M, Demerath RR. Hypoxemia during Oral Feeding of Children with Severe Cerebral Palsy. Developmental Medicine and Child Neurology 1993;35:3-10.

13. Lanert G, Ekberg O. Positioning Improves the Oral and Pharyngeal Swallowing Function in Children with Cerebral Palsy. Acta Paediatr 1995;84:689-92

14.Jones PM. Feeding disorders in children with multiple handicaps. Developmental Medicine and Child Neurology jun 1989;31(3):404-6.

15. Reilly S, Skuse D, Poblete X. Prevalence of feeding problems and oral motor dysfunction in children with cerebral palsy: a community survey. The Journal of Pediatrics 1996;129:877-82. 
16. Tuchman DN. Physiology of the Swallowing Apparatus. In: Tuchman DN, Walter RS. (Eds.) Disorders of Feeding and Swallowing in Infants and Children: Pathophysiology, Diagnosis, and Treatment. San Diego California: Singular Publishing Group 1994:1-25.

17. Wright RER, Wright FR, Carson CA. Videofluoroscopic Assessment in Children with Severe Cerebral Palsy Presenting with Dysphagia. Pediatr Radiol 1996;26:720-722.

18. Park RHR, Allison MC, Lang J et al. Randomised Comparison of Percutaneous Endoscopic Gastrostomy and Nasogastric Tube Feeding in Patients with Persisting Neurological Dysphagia. BMJ May 1992;304(6839):1406-1409.
19. Tawfiik R, Dickson A, Clarke M, Thomas AG. Caregivers Perceptions Following Gastrostomy in Severely Disabled Children with Feeding Problems. Developmental Medicine and Child Neurology 1997;39:746-751.

20. Buchholz DW. Neurologic Disorders of Swallowing. In: Groher ME. Dysphagia: Diagnosis and Management $3^{\text {rd }}$ Edition, Boston: Butterworth-Heinemann, 1997:37-72.

21. Gisel EG, Applegate-Ferrante T, Benson J, Bosma JF. Oral-Motor Skills Following Sensoriomotor Therapy in Two Groups of Moderately Dysphagic Children with Cerebral Palsy: Aspiration vs Nonaspiration. Dysphagia spring 1996;11:59-71.

22. Helfrich-Miller KR, Rector KL, Straka JA. Dysphagia: its Treatment in the Profoundly Retarded Patient with Cerebral Palsy. Arch Phys Med Rehabil Aug 1986;67(8):520-5. 